Supplement of Hydrol. Earth Syst. Sci., 21, 3991-4010, 2017

https://doi.org/10.5194/hess-21-3991-2017-supplement

(c) Author(s) 2017. This work is distributed under

the Creative Commons Attribution 3.0 License.

(c) (1)

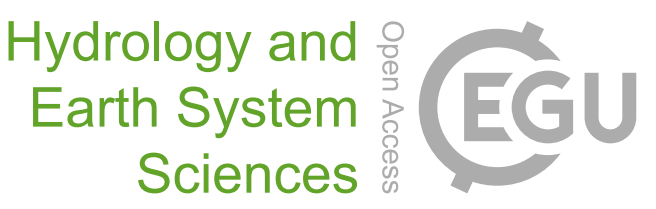

Supplement of

\title{
Has dyke development in the Vietnamese Mekong Delta shifted flood hazard downstream?
}

Nguyen Van Khanh Triet et al.

Correspondence to: Nguyen Van Khanh Triet (triet@gfz-potsdam.de)

The copyright of individual parts of the supplement might differ from the CC BY 3.0 License. 
Table S1. List of gauged data used in this study, name of station, its location, type of data and purpose of usage

\begin{tabular}{|c|c|c|c|c|c|c|c|}
\hline \multirow[t]{2}{*}{ no. } & \multirow{2}{*}{$\begin{array}{c}\text { name of } \\
\text { station }\end{array}$} & \multicolumn{3}{|c|}{ geographical location } & \multicolumn{3}{|c|}{$\overline{\text { Data }}$} \\
\hline & & lat. & long. & located at & data & type of data & used in \\
\hline 1 & Kratie & $12.260^{\circ} \mathrm{N}$ & $105.984^{\circ} \mathrm{E}$ & Mekong river (CFP) & ${\mathrm{H} \& \mathrm{Q}^{1}}^{2}$ & daily & trend \& boundary \\
\hline 2 & $\begin{array}{l}\text { Kampong } \\
\text { Cham }\end{array}$ & $11.932^{\circ} \mathrm{N}$ & $105.336^{\circ} \mathrm{E}$ & Mekong river (CFP) & $\mathrm{H}$ & daily & calibration \\
\hline 3 & Prekdam & $11.815^{\circ} \mathrm{N}$ & $104.807^{\circ} \mathrm{E}$ & Tonlesap river (CFP) & $\mathrm{H}$ & daily & calibration \\
\hline 4 & $\begin{array}{l}\text { Phnom Penh } \\
\text { port }\end{array}$ & $11.583^{\circ} \mathrm{N}$ & $104.924^{\circ} \mathrm{E}$ & Tonlesap river (CFP) & $\mathrm{H}$ & daily & calibration \\
\hline 5 & Neakluong & $11.260^{\circ} \mathrm{N}$ & $105.280^{\circ} \mathrm{E}$ & Mekong river (CFP) & $\mathrm{H}$ & daily & calibration \\
\hline 6 & Khokhel & $11.255^{\circ} \mathrm{N}$ & $105.030^{\circ} \mathrm{E}$ & Bassac river (CFP) & $\mathrm{H}$ & daily & calibration \\
\hline 7 & Tan Chau & $10.805^{\circ} \mathrm{N}$ & $105.235^{\circ} \mathrm{E}$ & Mekong river (VMD) & H\&Q & daily/hourly & trend \& calibration \\
\hline 8 & Chau Doc & $10.709^{\circ} \mathrm{N}$ & $105.125^{\circ} \mathrm{E}$ & Bassac river (VMD) & $H \& Q$ & daily/hourly & trend \& calibration \\
\hline 9 & Vam Nao & $10.567^{\circ} \mathrm{N}$ & $105.350^{\circ} \mathrm{E}$ & Vam Nao river (VMD) & $H \& Q$ & hourly & calibration \\
\hline 10 & Long Xuyen & $10.393^{\circ} \mathrm{N}$ & $105.435^{\circ} \mathrm{E}$ & Bassac river (VMD) & $\mathrm{H}$ & hourly & calibration \\
\hline 11 & Cao Lanh & $10.417^{\circ} \mathrm{N}$ & $105.644^{\circ} \mathrm{E}$ & Mekong river (VMD) & $\mathrm{H}$ & hourly & calibration \\
\hline 12 & Can Tho & $10.041^{\circ} \mathrm{N}$ & $105.799^{\circ} \mathrm{E}$ & Bassac river (VMD) & H\&Q & daily/hourly & trend \& calibration \\
\hline 13 & My Thuan & $10.278^{\circ} \mathrm{N}$ & $105.910^{\circ} \mathrm{E}$ & Mekong river (VMD) & $H \& Q$ & daily/hourly & trend \& calibration \\
\hline 14 & Hung Thanh & $10.659^{\circ} \mathrm{N}$ & $105.779^{\circ} \mathrm{E}$ & Phuoc Xuyen canal (VMD) & $\mathrm{H}$ & hourly & calibration \\
\hline 15 & Moc Hoa & $10.777^{\circ} \mathrm{N}$ & $105.935^{\circ} \mathrm{E}$ & Vaico river (VMD) & $\mathrm{H}$ & hourly & calibration \\
\hline 16 & Kien Binh & $10.617^{\circ} \mathrm{N}$ & $106.050^{\circ} \mathrm{E}$ & Kenh 12 canal (VMD) & $\mathrm{H}$ & hourly & calibration \\
\hline 17 & Xuan To & $10.606^{\circ} \mathrm{N}$ & $104.944^{\circ} \mathrm{E}$ & Vinh Te canal (VMD) & $\mathrm{H}$ & hourly & calibration \\
\hline 18 & Tri Ton & $10.436^{\circ} \mathrm{N}$ & $105.056^{\circ} \mathrm{E}$ & Tri Ton canal (VMD) & $\mathrm{H}$ & hourly & calibration \\
\hline 19 & Tan Hiep & $10.118^{\circ} \mathrm{N}$ & $105.285^{\circ} \mathrm{E}$ & Cai San canal (VMD) & $\mathrm{H}$ & hourly & calibration \\
\hline 20 & Vi Thanh & $9.784^{\circ} \mathrm{N}$ & $105.467^{\circ} \mathrm{E}$ & Xa No canal (VMD) & $\mathrm{H}$ & hourly & calibration \\
\hline 21 & Vung Tau & $10.333^{\circ} \mathrm{N}$ & $107.067^{\circ} \mathrm{E}$ & South China Sea & $\mathrm{H}$ & hourly & model boundary \\
\hline 22 & Vam Kenh & $10.270^{\circ} \mathrm{N}$ & $106.740^{\circ} \mathrm{E}$ & $\begin{array}{l}\text { Tieu branch - Mekong } \\
\text { estuary }\end{array}$ & $\mathrm{H}$ & hourly & model boundary \\
\hline 23 & Binh Dai & $10.197^{\circ} \mathrm{N}$ & $106.711^{\circ} \mathrm{E}$ & $\begin{array}{l}\text { Dai branch - Mekong } \\
\text { estuary }\end{array}$ & $\mathrm{H}$ & hourly & model boundary \\
\hline 24 & An Thuan & $9.976^{\circ} \mathrm{N}$ & $106.605^{\circ} \mathrm{E}$ & $\begin{array}{l}\text { Ham Luong branch - } \\
\text { Mekong estuary }\end{array}$ & $\mathrm{H}$ & hourly & model boundary \\
\hline 25 & Ben Trai & $9.881^{\circ} \mathrm{N}$ & $106.529^{\circ} \mathrm{E}$ & $\begin{array}{l}\text { Co Chien branch - Mekong } \\
\text { estuary }\end{array}$ & $\mathrm{H}$ & hourly & model boundary \\
\hline 26 & My Thanh & $9.425^{\circ} \mathrm{N}$ & $106.171^{\circ} \mathrm{E}$ & Bassac estuary & $\mathrm{H}$ & hourly & model boundary \\
\hline 27 & Ganh Hao & $9.031^{\circ} \mathrm{N}$ & $105.419^{\circ} \mathrm{E}$ & Ganh Hao estuary & $\mathrm{H}$ & hourly & model boundary \\
\hline 28 & Song Doc & $9.041^{\circ} \mathrm{N}$ & $104.833^{\circ} \mathrm{E}$ & Song Doc river (VMD) & $\mathrm{H}$ & hourly & model boundary \\
\hline 29 & Xeo Ro & $9.865^{\circ} \mathrm{N}$ & $105.111^{\circ} \mathrm{E}$ & Cai Lon estuary (VMD) & $\mathrm{H}$ & hourly & model boundary \\
\hline 30 & Rach Gia & $10.012^{\circ} \mathrm{N}$ & $105.084^{\circ} \mathrm{E}$ & Gulf of Thailand & $\mathrm{H}$ & hourly & model boundary \\
\hline
\end{tabular}

${ }^{1} H$ - water stage data, $Q$ - discharge data 
Table S2. Changes in inundation area as percentage of provincial area between the two model setups (with / without high-dyke system). The numbers are the mean of the two model setups as given in Table 3 .

\begin{tabular}{|c|c|c|c|c|c|c|c|c|c|c|}
\hline \multirow[t]{2}{*}{ No. } & \multirow[t]{2}{*}{ Province } & \multicolumn{3}{|c|}{$\begin{array}{l}\text { no high-dyke systems } \\
\text { (Scenarios S1 \& S3) }\end{array}$} & \multicolumn{3}{|c|}{$\begin{array}{l}\text { with high-dyke systems } \\
\text { (Scenarios S2 \& S4) }\end{array}$} & \multicolumn{3}{|c|}{ changes } \\
\hline & & $\begin{array}{c}\text { shallow } \\
\text { inundation } \\
(<1.0 \mathrm{~m})\end{array}$ & $\begin{array}{c}\text { deep } \\
\text { inundation } \\
(>1.0 \mathrm{~m})\end{array}$ & $\begin{array}{c}\text { total } \\
\text { inundated } \\
\text { area }\end{array}$ & $\begin{array}{c}\text { shallow } \\
\text { inundation } \\
(<1.0 \mathrm{~m})\end{array}$ & $\begin{array}{c}\text { deep } \\
\text { inundation } \\
(>1.0 m)\end{array}$ & $\begin{array}{c}\text { total } \\
\text { inundated } \\
\text { area }\end{array}$ & $\begin{array}{c}\text { shallow } \\
\text { inundation } \\
(<1.0 \mathrm{~m})\end{array}$ & $\begin{array}{c}\text { deep } \\
\text { inundation } \\
(>1.0 m)\end{array}$ & $\begin{array}{c}\text { total } \\
\text { inundated } \\
\text { area }\end{array}$ \\
\hline 1 & $\begin{array}{l}\text { An } \\
\text { Giang }\end{array}$ & 5.4 & 73.0 & 78.5 & 3.0 & 43.8 & 46.8 & -2.4 & -29.2 & -31.7 \\
\hline 2 & $\begin{array}{l}\text { Dong } \\
\text { Thap }\end{array}$ & 13.1 & 59.4 & 72.5 & 5.7 & 48.1 & 53.8 & -7.4 & -11.4 & -18.7 \\
\hline 3 & $\begin{array}{l}\text { Long } \\
\text { An }\end{array}$ & 37.9 & 36.3 & 74.2 & 39.3 & 38.1 & 77.4 & 1.4 & 1.8 & 3.2 \\
\hline 4 & $\begin{array}{l}\text { Kien } \\
\text { Giang }\end{array}$ & 33.2 & 22.5 & 55.7 & 31.9 & 23.8 & 55.7 & -1.3 & 1.3 & 0.0 \\
\hline 5 & $\begin{array}{l}\text { Can } \\
\text { Tho }\end{array}$ & 37.3 & 34.8 & 72.1 & 30.7 & 41.4 & 72.1 & -6.6 & 6.6 & 0.0 \\
\hline 5 & $\begin{array}{l}\text { Vinh } \\
\text { Long }\end{array}$ & 51.7 & 35.7 & 87.4 & 39.6 & 48.0 & 87.7 & -12.0 & 12.3 & 0.3 \\
\hline 7 & $\begin{array}{l}\text { Hau } \\
\text { Giang }\end{array}$ & 42.1 & 21.6 & 63.7 & 43.5 & 24.5 & 68.0 & 1.5 & 2.9 & 4.4 \\
\hline 8 & $\begin{array}{l}\text { Tien } \\
\text { Giang }\end{array}$ & 41.9 & 14.7 & 56.6 & 41.5 & 18.0 & 59.5 & -0.3 & 3.3 & 2.9 \\
\hline 9 & $\begin{array}{l}\text { Ben } \\
\text { Tre }\end{array}$ & 51.3 & 29.0 & 80.3 & 52.0 & 29.4 & 81.4 & 0.6 & 0.5 & 1.1 \\
\hline
\end{tabular}

